\title{
Unravelling the genetic components involved in the immune response of pigs vaccinated against influenza virus
}

\author{
Ricardo Zanella ${ }^{\mathrm{a}, \mathrm{b}, 1}$, Danielle Gava ${ }^{\mathrm{a}, 1}$, Jane de Oliveira Peixoto ${ }^{\mathrm{a}}$, Rejane Schaefer ${ }^{\mathrm{a}}$, \\ Janice Reis Ciacci-Zanella ${ }^{a}$, Natalha Biondo ${ }^{c}$, Marcos Vinicius Gualberto Barbosa da Silva ${ }^{d}$, \\ Maurício Egídio Cantão ${ }^{\mathrm{a}}$, Mônica Corrêa Ledur ${ }^{\mathrm{a}, *}$ \\ a Embrapa Swine and Poultry, Concórdia, SC, Brazil \\ b Present Address: University of Passo Fundo, Passo Fundo, RS, Brazil \\ c Santa Catarina State University, Lages, SC, Brazil

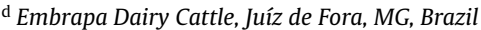

\section{A R T I C L E I N F O}

\section{Article history:}

Received 29 April 2015

Received in revised form 31 August 2015

Accepted 4 September 2015

Available online 8 September 2015

\section{Keywords:}

GWAS

Swine

Influenza A virus

Vaccination

Immune response

\begin{abstract}
A B S T R A C T
A genome-wide association study for immune response to influenza vaccination in a crossbred swine population was conducted. Swine influenza is caused by influenza A virus (FLUAV) which is considered one of the most prevalent respiratory pathogens in swine worldwide. The main strategy used to control influenza in swine herds is through vaccination. However, the currently circulating FLUAV subtypes in swine are genetically and antigenically diverse and their interaction with the host genetics poses a challenge for the production of efficacious and cross-protective vaccines. In this study, 103 pigs vaccinated with an inactivated H1N1 pandemic virus were genotyped with the Illumina PorcineSNP60V2 BeadChip for the identification of genetic markers associated with immune response efficacy to influenza A virus vaccination. Immune response was measured based on the presence or absence of HA (hemagglutinin) and NP (nucleoprotein) antibodies induced by vaccination and detected in swine sera by the hemagglutination inhibition (HI) and ELISA assays, respectively. The ELISA test was also used as a measurement of antibody levels produced following the FLUAV vaccination. Associations were tested with $x^{2}$ test for a case and control data and using maximum likelihood method for the quantitative data, where a moderate association was considered if $p<5 \times 10^{-5}$. When testing the association using the HI results, three markers with unknown location and three located on chromosomes SSCX, SSC14 and SSC18 were identified as associated with the immune response. Using the response to vaccination measured by ELISA as a qualitative and quantitative phenotype, four genomic regions were associated with immune response: one on SSC12 and three on chromosomes SSC1, SSC7, and SSC15, respectively. Those regions harbor important functional candidate genes possibly involved with the degree of immune response to vaccination. These results show an important role of host genetics in the immune response to influenza vaccination. Genetic selection for pigs with better response to FLUAV vaccination might be an alternative to reduce the impact of influenza virus infection in the swine industry. However, these results should to be validated in additional populations before its use.
\end{abstract}

(C) 2015 Elsevier B.V. All rights reserved.

\section{Introduction}

In the livestock sector, most of the economic losses are caused by infectious pathogens (Miller et al., 2013). To mitigate those losses,

\footnotetext{
* Corresponding author at: Embrapa Swine and Poultry, Animal Health and Genetics Laboratory. BR 153, Km 110, Vila Tamanduá 89700-000, Concórdia, Santa Catarina, Brazil. Fax: +55 4934410497

E-mail address: monica.ledur@embrapa.br (M.C. Ledur).

1 These authors contributed equally to this work.
}

several strategies have been proposed and implemented. Currently, vaccination is considered the most powerful tool to control and to diminish the disease burden, reducing the clinical signs and the transmission of the disease within and across herds (Kimman et al., 2007). However, the immune response to vaccination can vary among individuals, with animals not responding at all or with different levels of response (Poland et al., 2014; Rajao et al., 2014).

It has been proposed that the immune response to vaccination is inherited as a complex quantitative trait with variation resulting from environmental and genetic factors (Newport et al., 2004). 
Some of the differences in the immune response to vaccination can be attributed to several factors other than the environmental ones, such as the vaccine itself, the vaccination procedure, and the ability of the host to respond to the immunization, which can be determined by sex, genetics, and age. Stressors and nutrition are likely to have a huge impact on the efficacy of the vaccine (Van Loveren et al., 2001). In addition, the induction of protective immunity generally requires the administration of multiple doses of vaccines, which can be labor intensive and expensive (Mitchell et al., 2013). Furthermore, it is known that not all animals are equally immunized in a commercial system. Therefore, better strategies to protect animals are needed (Meeusen et al., 2007).

Influenza A virus (FLUAV) is a zoonotic pathogen that causes an acute respiratory infection in humans and other animal species, and it is also considered a global health concern. The FLUAV infection in swine causes major economic impact in most pig-producing countries around the world due to the high morbidity observed in affected farms (Bennett, 2003; Bennett and Ijpelaar, 2005; Olsen et al., 2002). In Brazil, swine influenza virus is currently one of the most important pathogens of the porcine respiratory disease complex, and genetically diverse FLUAV subtypes (H1N1pdm, H1N2, and $\mathrm{H} 3 \mathrm{~N} 2$ ) are circulating in pig farms in most of the Brazilian states (Nelson et al., 2015; Rajao et al., 2013; Schaefer et al., 2011; Schaefer et al., 2015). Moreover, no commercial FLUAV vaccine was available in Brazil until July of 2014 (Schaefer et al., 2015).

Swine influenza vaccines are commonly used in commercial herds in several countries to control the clinical signs of the disease (Rajao et al., 2014). However, the effectiveness of the vaccines may vary in pig herds and multiple factors, as for example, the lack of cross-protective immunity due to the high genetic variability of FLUAVs, might hinder their success in controlling influenza outbreaks (Rajao et al., 2014). Several studies were conducted to evaluate the efficacy of different types of vaccines to FLUAV in a swine population showing different levels of immune response (Hoft et al., 2011; Masic et al., 2010; Sandbulte et al., 2014). Despite concerns regarding the prevention and transmission of swine influenza virus, no studies have investigated the interaction between the host genetics and the immune response to influenza vaccination. Therefore, understanding the genetic mechanisms involved with the immune response to FLUAV vaccination will contribute to the development of new methods to improve the vaccination efficacy, reducing and preventing the transmission of FLUAV within pig herds. Therefore, the objective of this study was to identify genetic markers associated with immune response to influenza vaccination in an experimental condition. To our knowledge, this is the first study evaluating the interaction of host genetics and the immune response to influenza vaccination in a swine population.

\section{Material and methods}

This study was conducted at Embrapa (Brazilian Agricultural Research Corporation) Swine and Poultry, Santa Catarina State, Brazil and had the approval of the Institutional Animal Care and Use Committee for all experimental protocols used (CEUA/CNPSA 004/2011).

\subsection{Experimental population}

One hundred and three piglets $(n=103,55$ females and 48 males) from three unrelated sires MS-115 (composite terminal sires developed by Embrapa Swine and Poultry) and 46 Landrace $\times$ Large White sows, with parity order ranging from one to six, were used in this study (Fig. 1). All sows were partially related to each other with an average identical-by-descent (IBD) of 0.18 (0.05-0.63).
The sows were kept in a group and moved to an individual farrowing crate ten days prior the farrowing. No cross fostering was performed with the experimental piglets to avoid interference of the maternal genetics and colostrum intake. After weaning, piglets were moved to a group-housing with seven piglets per pen. The grouped animals were kept together until the end of the experiment.

\subsection{FLUAV vaccine preparation and vaccination of piglets}

Influenza vaccine was prepared at Embrapa Swine and Poultry, using the virus strain A/swine/Brazil/107-3A/2010 (H1N1) (Genbank accession number KF683611-KF683618) isolated from piglets in southern Brazil in 2010, and genetically characterized as H1N1pdm virus (Schaefer et al., 2015). The virus antigen was propagated in Madin-Darby canine kidney (MDCK) cells at approximately 64HA units or $10^{4.38} 50 \%$ tissue culture infectious dose $\left(\mathrm{TCID}_{50}\right)$ per $\mathrm{mL}$, as calculated by the Reed and Muench method (Reed and Muench, 1938). The virus inactivation was performed using a binary ethyleneimine (BEI) (Sigma-Aldrich). A commercial oil-in-water adjuvant (Emulsigen D, MVP Labs) at a v:v ratio of 4:1 virus to adjuvant was added to the inactivated virus, as described by Gauger and colleagues (Gauger et al., 2011).

Piglets were vaccinated with $1.5 \mathrm{~mL}$ of this vaccine via intramuscular route (neck) at approximately five weeks of age (34 days-old) and boosted at eight weeks of age (55 days-old) with the same dose. The age of the first vaccination was chosen based on preliminary data from our group that indicated the absence of maternal derived antibody (MDA) at this time (data not shown). The sows were not vaccinated against FLUAV since no commercial vaccine was available in Brazil during the experimental study (Schaefer et al., 2015).

\subsection{Sample collection}

The experimental study was run from January to June of 2014. Blood samples were collected through jugular puncture from piglets at $21,34,55$, and 76 days-old and from sows at the weaning time for the detection of FLUAV antibodies (Fig. 1). Piglets body weight was measured at birth, weaning ( 21 days-old), and 21 days after the second vaccine dose (76 days-old). Lung tissue samples were collected from piglets during slaughter at 153 days-old and stored at $-80^{\circ} \mathrm{C}$. In addition, ear tissue samples from 32 out of the 46 sows were also collected for genotyping. Fourteen sows were not collected because they were culled right after weaning.

\subsection{Antibody detection assays}

Antibodies produced against the hemagglutinin (HA) and the nucleoprotein (NP) of FLUAV were measured in swine sera by hemagglutination inhibition (HI) assay and ELISA, respectively.

The virus antigen in the HI assay was the same used for the vaccine preparation (A/swine/Brazil/107-3A/2010(H1N1)), which was previously characterized in (Schaefer et al., 2015). For HI, sera were heat inactivated at $56^{\circ} \mathrm{C}$ for $30 \mathrm{~min}$, then treated with a $20 \%$ suspension of kaolin (Sigma-Aldrich), and adsorbed with $0.5 \%$ turkey red blood cells. The reciprocal of the highest serum dilution with a positive well was considered the HI titer. Antibody titers less than 10 were considered negative, titers between 10 and 20 were considered "suspect" and titers of 40 or greater were considered positive (Kitikoon et al., 2014).

The ELISA assay (Influenza A-Ab test; IDEXX ${ }^{\circledR}$ Laboratories Inc.) uses a monoclonal antibody generated against human influenza A virus subtype H1N1 and recognizes a highly conserved epitope of influenza A nucleoprotein (NP). Sample-to-negative (S/N) ratio was calculated according to the manufacturer instructions. Samples 
ELISA ELISA

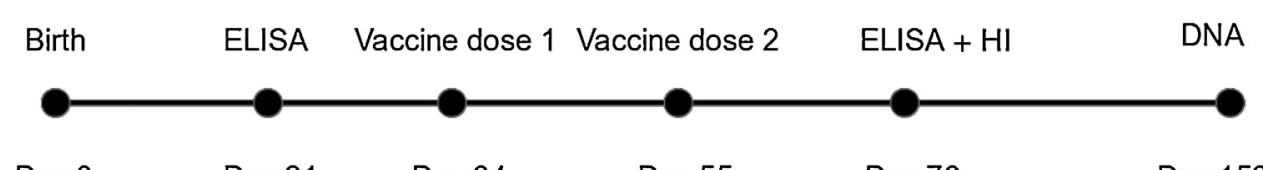

$\begin{array}{lllll}\text { Day } 0 & \text { Day } 21 & \text { Day } 34 & \text { Day } 55 & \text { Day } 76\end{array}$

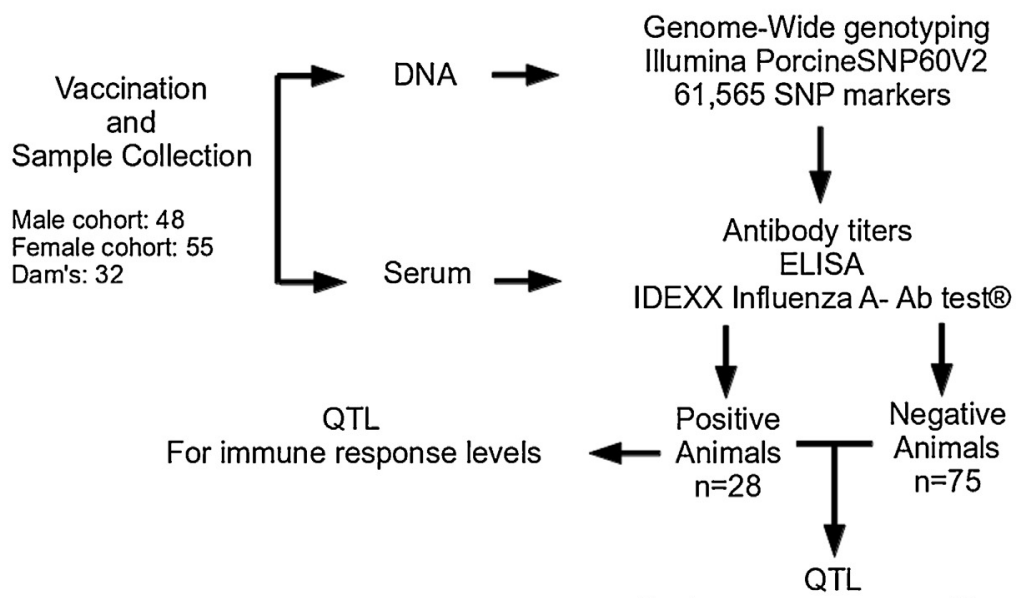

For immune response efficacy

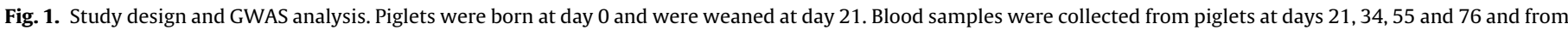

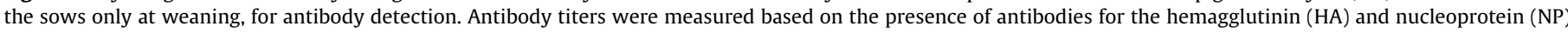

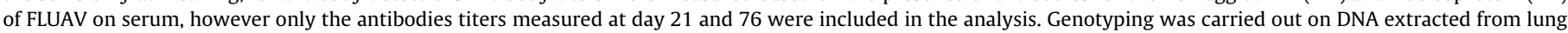

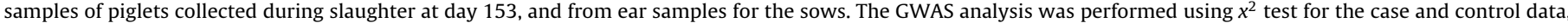
and a linear mixed model using a Maximum Likelihood method to test SNP association with the quantitative measurement of immune response.

were considered positive if an $\mathrm{S} / \mathrm{N}$ ratio was $\leq 0.60$. The sensitivity of this test is $95.5 \%$ and its specificity is $99.6 \%$, being considered an accurate indicator of the individual infection status (Goodell et al., 2014).

\subsection{Genomic DNA extraction and SNP genotyping}

Genomic deoxyribonucleic acid (DNA) extraction was performed on $200 \mathrm{mg}$ of tissue (lung or ear tissue samples), using PureLink $^{\circledR}$ Genomic DNA Mini Kit (Invitrogen), according to the manufacturer instructions. The quantity and quality of DNA were assessed using $260 / 280 \mathrm{~nm}$ wavelength readings from a NanoDrop ND-2000 spectrophotometer (NanoDrop Technologies Inc.). The $260 / 280 \mathrm{~nm}$ readings for all samples ranged between 1.8 and 2.0. The DNA was subsequently diluted to $50 \mathrm{ng} / \mu \mathrm{L}$ in $10 \mu \mathrm{L}$ and genotyped at Deoxi Biotecnologia Brazil with the Illumina PorcineSNP60V2 BeadChip whole-genome single nucleotide polymorphism (SNP) assay, which contains 61,565 SNPs across the swine genome.

\subsection{Evaluated phenotypes}

\subsubsection{Maternal derived antibody (MDA)}

Maternally derived antibodies were measured based on the levels of antibody transmitted to the offspring via sow colostrum. Piglets were classified as positive or negative based on the $\mathrm{S} / \mathrm{N}$ ratio due to the presence or absence of NP antibody. This was directly related to MDA via colostrum intake, since none of the piglets from the negative sows presented antibodies to FLUAV. Regarding the sows antibodies, those reflect a natural infection, once vaccination was not available in Brazil at that time. To test the effect of sow genetics on the MDA in piglets, a transmission disequilibrium test (TDT) with the piglets and dams $\mathrm{S} / \mathrm{N}$ ratio information at weaning time (21 days-old) was used.

\subsubsection{Acquired immunity}

The acquired immunity was evaluated based on the piglet antibody production directly related to the vaccination, since a nonvaccinated group was raised with vaccinated pigs as an indicator of possible circulating FLUAV that could cause a natural infection (data not shown). Piglets were classified as positive or negative, based on the presence or absence of antibodies against HA and NP proteins following vaccination. For both tests, the piglets immune status were measured 21 days after the second vaccine dose (76 days-old).

\subsection{Data quality control}

Data quality control was performed using PLINK V1.9 (Purcell et al., 2007). Samples were removed from the analysis if more than $10 \%$ of their genotypes failed, and if they had paternity inconsistency. To evaluate inconsistency among individuals, the degree of relationship between animals was estimated based on the genomic segments shared IBD and results were compared with pedigree relationship information.

The quality of SNPs was assessed prior the analysis. SNPs were removed if they had a Minor Allele Frequency (MAF) $<1 \%$ or if they failed in more than $10 \%$ of the samples. To test for population stratification among animals used in this study, a multi-dimensional scaling (MDS) plot was constructed using PLINK (Purcell et al., 2007) and the R statistical environment (Team, 2009). Of the 61,565 SNPs, only 5180 were not in linkage disequilibrium (LD), $r^{2}<0.2$, and therefore were used for the MDS plot construction. PLINK was also used to construct the QQ-plot and to compute the genomic inflation factor $\left(\lambda_{G C}\right)$ to test for population substructure.

\subsection{Statistical analysis}

A Genome-Wide Association Study (GWAS) was conducted to identify loci associated with immune response to influenza 


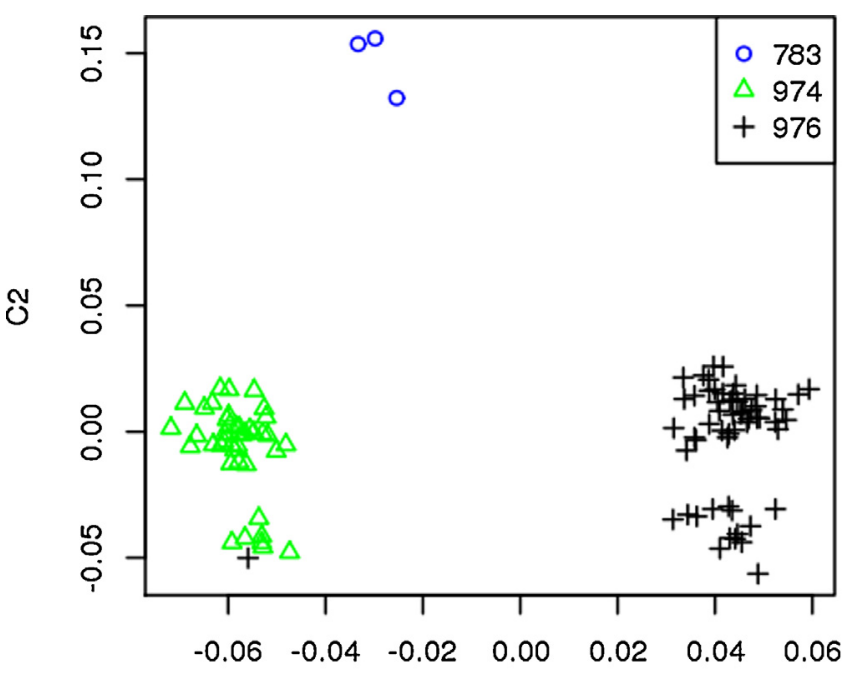

C1

Fig. 2. Multidimensional scaling plot (MDS Plot). The MDS plot provides a spatial representation of data that can facilitate interpretation and reveals structural relationships in the populations used in this study. Blue circles represent animals out of sire 783 , black crosses are animals out of sire 976 , and green triangles are animals out of sire 974. In this experiment, the MDS plot identified one animal with incorrect paternity.

vaccination. The TDT was performed to evaluate the maternal genetic effects on the MDA of the littermate using PLINK (Purcell et al., 2007). For the acquired immunity, a case and control analysis was performed using a standard chi-square test and the odds ratio was computed for the favorable allele for each marker using PLINK.

From the ELISA results, positive pigs were further classified based on the quantity of antibody ( $\mathrm{S} / \mathrm{N}$ ratio) produced by their immune system and submitted to a quantitative statistical test. Data were analyzed with a linear mixed model using a Maximum Likelihood method in the QxPaK 5.05 program (Perez-Enciso and Misztal, 2011). The model included the fixed effects of sex, parity order and SNP, and animal and residual as random effects. The piglet birth weight and weight at vaccination were used as covariates. The additive (a) effect of each SNP was tested. Heritability was estimated by the Restricted Maximum Likelihood method with the QxPaK program using the same mixed model described previously.

Markers were considered moderated associated if an unadjusted $p$-value was $<5.0 \times 10^{-5}$, based on the Wellcome Case and Control Consortium recommendations (Wellcome Trust Case Control, 2007). Physical position of each SNP was expressed relative to the forward strand of the reference genome (Sscrofa 10.2) (Badke et al., 2013). Putative positional candidate genes within $200 \mathrm{~kb}$ of the associated SNPs with immune response to FLUAV were searched in the NCBI and UCSC databases.

\section{Results}

\subsection{Antibody detection by ELISA}

An ELISA test was used as an indicator of the humoral immune status of the herd, MDA decay, vaccine humoral immune response and host exposure to FLUAV. At weaning time (21 days-old), nineteen sows $(n=19)$ had FLUAV antibodies caused by natural infection and therefore, were considered responders and twenty-seven sows $(n=27)$ did not have FLUAV antibodies and were classified as non-responders. Out of the responder sows, fifteen $(n=15)$ were genotyped, and seventeen $(n=17)$ of the non-responders sows were also genotyped. For the piglets at weaning, 26 had FLUAV antibodies, possibly related to MDA, since they were from positive sows, and seventy-seven piglets were negative.

At the day of the first vaccination (34 days-old), none of the piglets used in this study had antibodies against FLUAV, indicating that any titer post-vaccination would be directly related to the immunization with our in house vaccine. The second vaccine dose was administered at 55 days-old and antibodies were measured at 76 days-old. Seventy-five animals $(n=75)$ did not respond to vaccination and therefore were classified as non-responders and twenty-eight animals $(n=28)$ responded to the vaccination and were considered as responders. In addition, the non-vaccinated group, which was raised with vaccinated piglets, was maintained negative, ensuring that FLUAV was not circulating in the herd during the course of the experiment. No correlation was identified between the presence or absence of piglets antibody from colostrum and acquired immunity $\left(r^{2}=-0.09, p=0.42\right)$.

\subsection{Antibody detection by $\mathrm{HI}$}

The HI test was used to measure anti-HA antibodies at 21 days after the second dose of the FLUAV vaccine (76 days of age). Fortysix pigs were negative by $\mathrm{HI}$, therefore they were classified as non-responders, as well as 38 animals with HI titers of $1: 10$ or $1: 20$. Piglets were considered to be protected if they have HI titers $\geq 1: 40$. Therefore, 19 pigs were classified as responders, with $\mathrm{HI}$ titers ranging from $1: 40$ to $1: 160$.

\subsection{Sample and SNP quality assurance}

One out of 103 samples was removed from the analysis due to a paternity problem (Fig. 2). Out of 61,565 SNPs from the array, 1489 SNPs were removed, because they failed in more than $10 \%$ of the samples, 5849 were removed due to low MAF and 2442 SNPs failed in both tests. After removing poor quality SNPs, 51,785 markers remained for this study. From those, 2197 SNPs were also eliminated because they failed the Hardy-Weinberg Equilibrium (HWE) test at a $p<9.65 \times 10^{-7}$. At the end, 49,588 SNPs with genotyping call rate of 0.99 in the remaining individuals were used for the association test. The QQ-plot did not show any indication of population stratification (Fig. 3a,b,c). The genomic inflation factor was $\lambda_{\mathrm{GC}}=1.2$ for $\mathrm{HI}, 1.1$ and 1 , for the ELISA quantitative and qualitative test, respectively, and $\lambda_{\mathrm{GC}}=1.03$ for the TDT test. These low levels of inflation indicate the absence of population substructure and do not require any adjustment to the statistical model used in the analysis.

\subsection{Transmission disequilibrium test}

A family based association test was conducted to verify the influence of the sow genetics on the MDA by the offspring via colostrum and measured by ELISA. No associated region was identified with the passive immunity, suggesting a stronger role of the antibodies from the colostrum to stimulate an immune response to influenza instead of a maternal genetic component. However, this result should be carefully interpreted since we had few animals per group. Twenty-six piglets from positive sows were positive at weaning. On the other hand, 16 piglets also from positive sows were negative at weaning. This was possibly due to the amount of colostrum intake during the first hours of life. All the negative piglets $(n=61)$ were from negative sows and were also negative at weaning. None of the negative sows have produced a positive piglet.

\subsection{GWAS with response to influenza vaccination}

A GWAS was performed with the presence or absence of antibodies against FLUAV detected in swine sera 
a

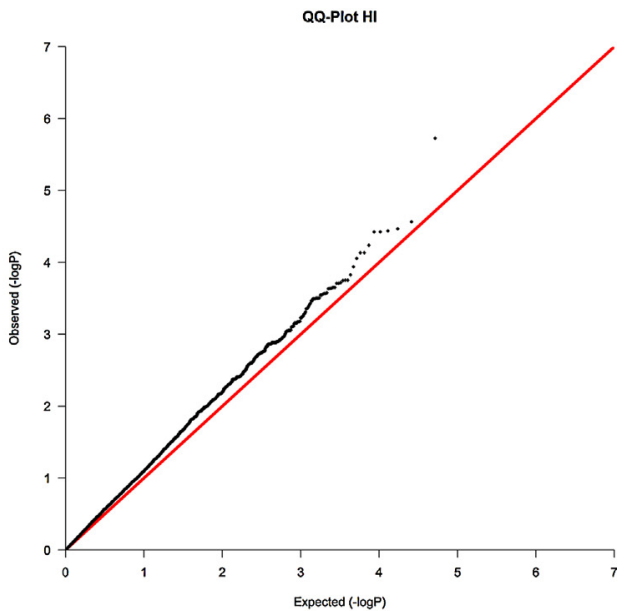

b

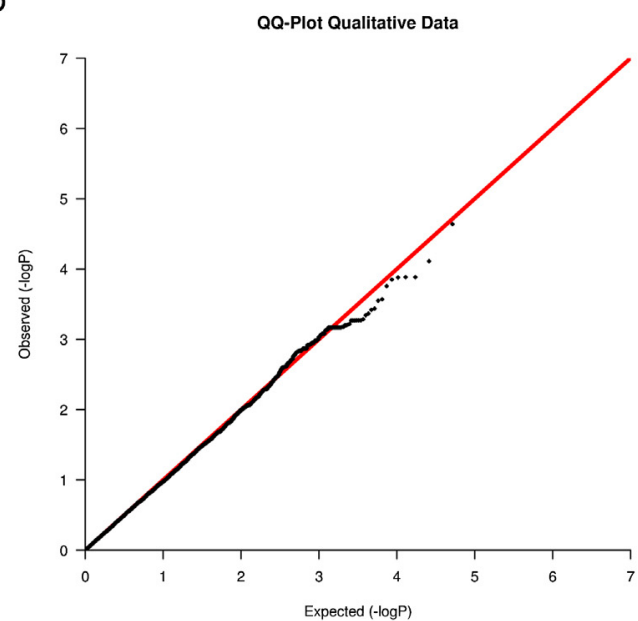

C

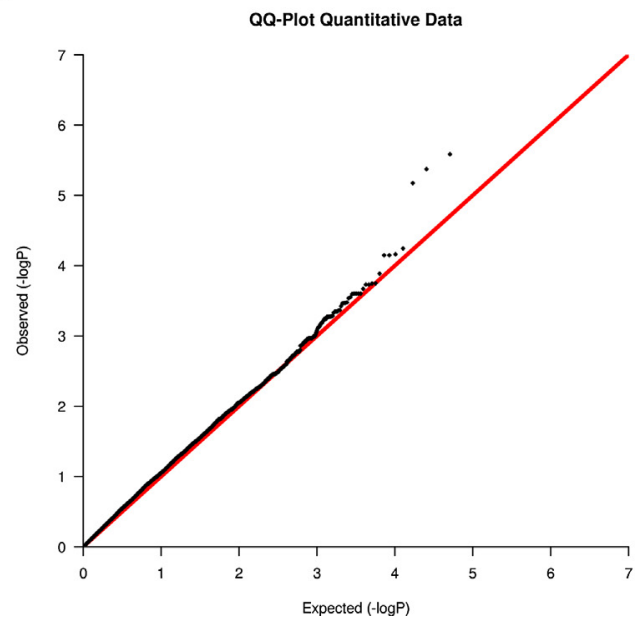

$\operatorname{SSC14}\left(p=3.7 \times 10^{-5}\right)$, and $r s 80970341$ located on SSCX position $135,519,159 \mathrm{bp}\left(p=3.4 \times 10^{-5}\right)$. In addition, three markers with unknown location were also associated with immune response: MARC0093463, rs81312872, and CAIL0000240, with $p=1.8 \times 10^{-6}, \quad p=3.6 \times 10^{-5}$ and $p=3.7 \times 10^{-5}$, respectively (Fig. 4a).

To test for associations of SNPs with immune response, using the presence of NP antibodies as a qualitative phenotype, pigs were classified as non-responders and responders. When we tested for this association, two markers were identified on SSC12: one located at $27,241,826 \mathrm{bp}$ and the other at $31,875,247 \mathrm{bp}$ of the pig genome, with moderate association $\left(p<5 \times 10^{-5}\right)$ with immune response (Fig. $4 \mathrm{~b}$ ). The LD between these two markers rs81433411 and $r s 81341245$ was $D^{\prime}=0.861$. The frequency of the "A" allele of rs81433411 was 0.58 in responders and 0.25 in non-responders. For the SNP $r s 81341245$, the frequency of the "G" allele was 0.31 in responders and 0.62 in non-responder animals. Testing the combined effect of the associated markers did not improve the significance of the test. For the haplotype composed of $r$ s81433411 and rs 81341245 , the GG haplotype had the frequency of 0.28 in responders and 0.60 in non-responder animals, and the AA haplotype had the frequency of 0.55 in responder and 0.22 in non-responder groups $\left(p<3.6 \times 10^{-5}\right)$.

The average ELISA test for all piglets $(n=103)$ was $0.76 \pm 0.24$ $(\min =0.22, \max =1.35)$. For the positive piglets $(n=28)$, the mean ELISA result was $0.45 \pm 0.12(\min =0.22, \max =0.59)$. Since the objective of our study was to identify genetic markers associated with immune response levels to influenza vaccination, just the positive animals were included in the quantitative analysis. The heritability estimate for the antibody response to vaccination using the positive animals was $h^{2}=0.37$. Analyzing the response to vaccination as a quantitative trait, three associated regions on chromosomes SSC1, SSC7, and SSC15 (Fig. 4c) and a marker rs80806920 with an unknown location were identified. Further analysis of the marker rs80806920 has shown a high LD $\left(D^{\prime}>0.9\right)$ with the associated region on SSC1. Exploring the marker rs80806920, we identified that its position based on Pig_GSeek80K (http:// bioinformatics.tecnoparco.org/SNPchimp/) is actually on SSC1 at $87,513,453 \mathrm{bp}$.

Eleven SNPs $\quad(r s 80867816, \quad r s 80791631, \quad r s 80864101$, rs80947054, rs81354872, DRGA0001165, DRGA0001172, DRGA0001218, rs342866101, MARC0022047, and MARC0037432) on SSC1:84,150,234-90,916,676, spanning a $6.7 \mathrm{Mb}$ region, were associated with the individual levels of antibodies produced $\left(p<5 \times 10^{-5}\right)$. The markers represent a single region based on high LD levels between all the associated SNPs in the region, averaging $D^{\prime}=0.96$. On SSC7, the marker MARC0078872, located at $48,375,311 \mathrm{bp}$, and the marker $r s 81332479$, on SSC15, located at $52,185,765 \mathrm{bp}$, were also associated with immune response levels with $p=2.9 \times 10^{-5}$ and $p=1.9 \times 10^{-5}$, respectively.

\section{Discussion}

Even with the best management practices, infectious diseases are still the most significant cause of economic losses to the animal industry. Therefore, new methodologies are needed to control and to reduce the losses caused by infectious pathogens. The understanding of the genetic mechanisms involved in the immune response to vaccination might provide new approaches to reduce the losses caused by infections. However, there are few studies on the interaction of host genetics with the immune response post-vaccination; even though there is considerable evidence that immune response is under genetic control (Glass, 2004). Most of the studies with animals have been concentrated on disease resis-

tance, susceptibility and tolerance (Kelm et al., 2001; Kich et al., post-vaccination. The phenotypes were obtained using the $\mathrm{HI}$ and ELISA results. Using the $\mathrm{HI}$ as a measurement of the immune response post FLUAV vaccination, significant associations were detected with markers rs81468495 located at $31,531,915 \mathrm{bp}$ on

SSC18 $\left(p=2.7 \times 10^{-5}\right)$, rs80880408 located at $79,101,636$ bp on
Fig. 3. Quantile-quantile plot(Q-Q plot). The Q-Q plot shows the observed p-values HI test; (b) ELISA qualitative test, and (c) ELISA quantitative test. The Q-Q plot shows little to no evidence of deviation from the expected null distribution of p-values and therefore shows no evidence of population substructure. Deviations towards the end of the graph indicate possible QTL existence. 
a

GWAS_HI

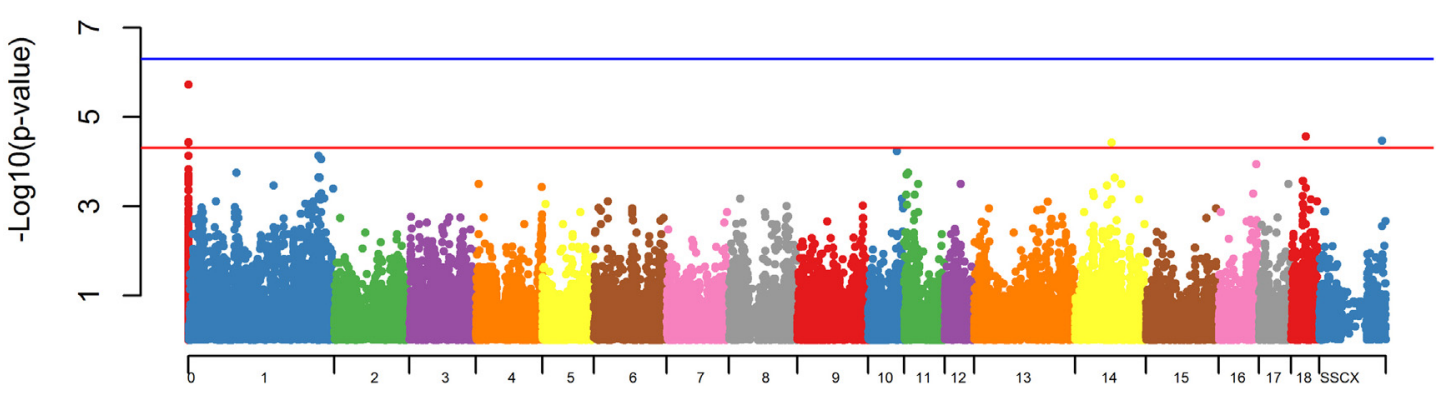

Chromosome

b

Immune Response Responders x Non-responders

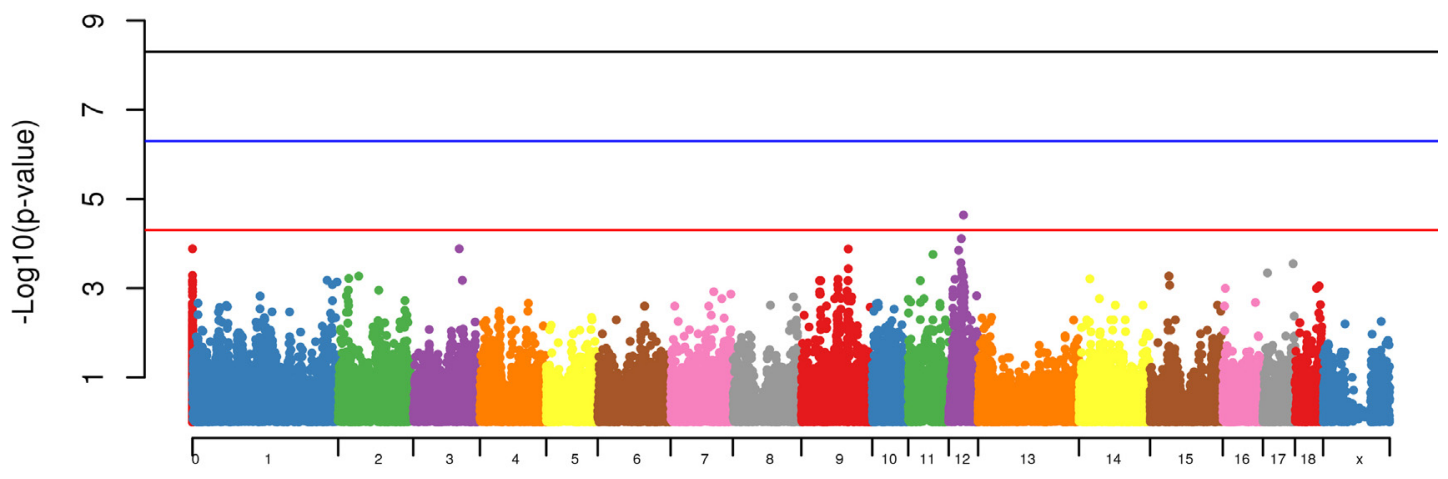

Chromosome

C

Immune Response Quantitative

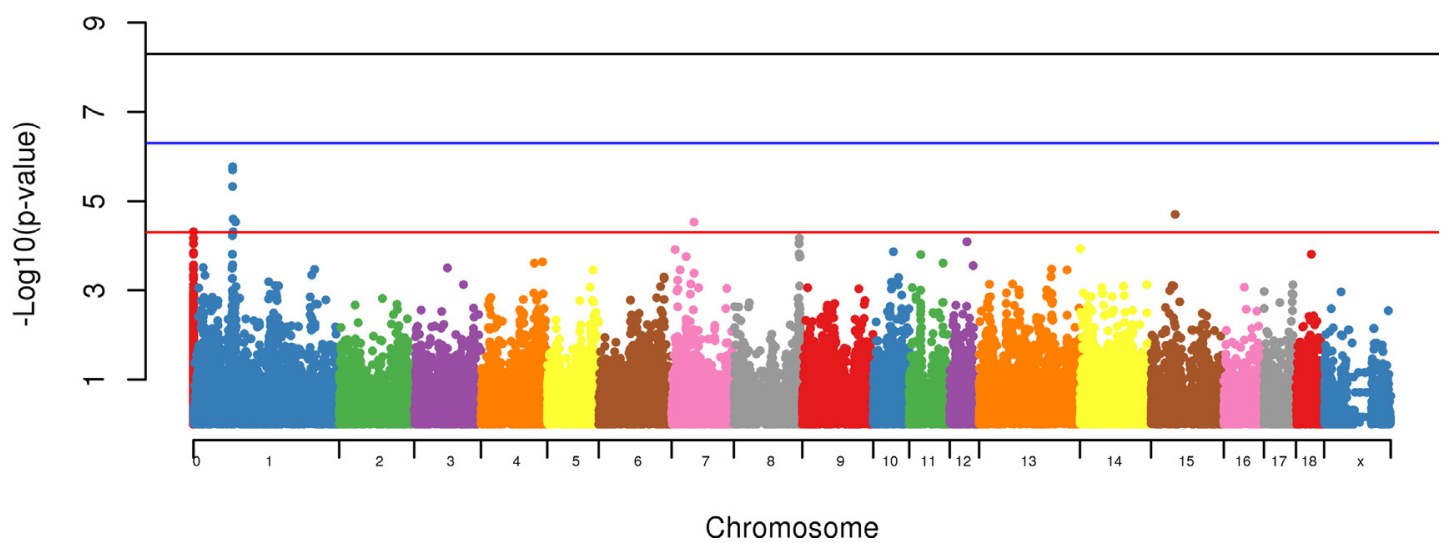

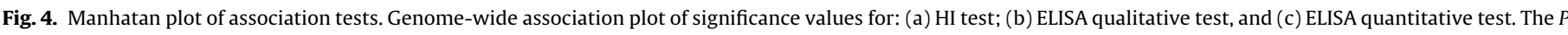

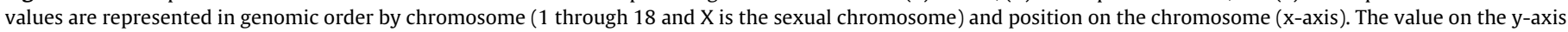
represents the $-\log _{10}$ of the p-value. SNPs located between 4.3 and 6.3 on the y axis provided moderated evidence for association.

2014; Settles et al., 2009; Zanella et al., 2011) and to a lesser extent on responsiveness to vaccination (Moreno et al., 2003; Sitte et al., 2002; Tan et al., 2001). These studies have identified important genetic components associated with the phenotypic variation for disease traits, with different heritability estimates. Another indication of the involvement of genetics in the response to vaccination is the variable level of antibodies produced by the individuals facing the same vaccination procedure. 
Detmer and colleagues (Detmer et al., 2013) have compared the effect of influenza infection in a group of piglets with high and low birth weight, and no statistical difference was observed between groups in total viral load and severity of lung lesions. In our study, the effect of body weight on the immune response to vaccination was also tested. However, no correlation was observed between animal birth weight or body weight at the time of vaccination and the animal immune response $(p>0.35)$, indicating that body weight is not a good parameter to identify which animals will have a better response to vaccination. In the present study, the efficacy of an in house oil-in-water inactivated vaccine to H1N1pdm FLUAV was evaluated and its interaction with host genetics was tested using two different approaches. In the first approach we tested the response to vaccination as a categorical trait to identify loci associated with animal immune status (responders or non-responders); in the second method we used the individual levels of antibodies produced by the positive animals in response to vaccination as a quantitative trait.

Detection of antibodies against FLUAV in swine sera following vaccination was carried out using two serological assays, HI and ELISA. HI assay is the gold standard for the detection of anti-HA serum antibodies and it is correlated to protection from clinical disease (Van Reeth et al., 2012). Therefore, the HI is the method of choice to evaluate the influenza vaccine efficacy. However, NP antibodies detected by ELISA have a role in the stimulation of both cell mediated and humoral immunity, therefore, contributing to the protective mechanism in vaccinated animals (Carragher et al., 2008). In addition, the non-vaccinated group did not seroconvert to FLUAV, indicating that the NP antibodies detected by ELISA in the sera of the responder pigs were induced by the influenza vaccination.

The seroconversion of the in house vaccine in pigs was $27.2 \%$ using ELISA and 18.4\% using HI. The different percentages found for the two serological tests may be due to the different sensitivities detected for each assay (Goodell et al., 2014). Besides, in humans, the protection level induced by the influenza vaccine can vary significantly from 10 to $60 \%$, according to the age and endpoints (CDC, 2015; Del Giudice and Rappuoli, 2015), or even less, as $15-23 \%$, particularly for children (CDC, 2015). Despite of that, the antigen dose, method of virus inactivation and adjuvant added to the vaccine preparation are determinant for its efficacy (Del Giudice and Rappuoli, 2015; Rajao et al., 2014).

In the present study, the low number of responding piglets was probably due to the final amount of antigen delivered by the vaccine, which was reduced because of the method used for virus inactivation. Nevertheless, it was possible to identify markers associated with immune response efficacy to influenza vaccination, possibly because of the large effect of the associated markers. The identified genetic association is in agreement with the high estimated heritability. However, this estimate needs to be considered carefully because of the small sample size used in this study.

Immune response is a complex biological process coordinated by the interaction of several factors including genetic components that control gene regulation and its expression. Protective immunity against infection with FLUAV involves both innate and acquired immune response. After infection, the innate immune response lead to the lysis of infected cells via natural killer cells or by the production of pro-inflammatory and chemostatic cytokines, such as the tumor necrosis factor $\alpha$ (TNF- $\alpha)$, IL-1 $\beta$, IL-6, and type I interferons (IFN) (Cox et al., 2004). The innate response signals the migration of cells as neutrophils, monocytes, macrophages and lymphocytes and subsequent stimulation of the adaptive immune system (Cox et al., 2004; Rajao et al., 2014). The acquired immune response to FLUAV includes mucosal and systemic humoral and cell-mediated immunity (CMI). The humoral immunity due to natural exposure or vaccination reduces or prevents subsequent FLUAV infection. The antibodies, mainly IgA and IgG, are directed against $\mathrm{HA}, \mathrm{NA}, \mathrm{M}$, and NP viral proteins, acting in the neutralization of viral infection, preventing or clearing the respiratory tract of FLUAV (Rajao et al., 2014). On the other hand, the CMI response includes CD4+T helper cells and $C D+8$ cytotoxic $T$ cells, playing a role in FLUAV clearance and recovery after natural infection (Larsen et al., 2000).

In this study, the transmission disequilibrium test was performed to verify the influence of the sow genetics on the transmission of maternal antibodies to the piglets. No direct association was found, indicating that the colostrum intake during the first hours of life is more important to the development of the piglet immunity than the genetic background of the sow.

The GWAS allowed the identification of several SNPs associated to the acquired immunity, based on the presence or absence of antibodies against HA and NP proteins induced by vaccination. Herein, several genomic regions harboring positional and functional candidate genes possibly involved with immune response regulation to FLUAV in swine were identified. Using the HI assay to measure the immune response induced by FLUAV vaccination, six markers were identified in the association analyses: three located at chromosomes SSC14, SSC18 and SSCX, and three markers with unknown location (MARC0093463, rs81312872, CAIL0000240).

In silico analysis was carried out to find functional candidate genes possibly involved with immune response regulation. When spanning a region of $100 \mathrm{~kb}$ of the associated marker on SSC14, two genes were identified: the GTP-binding protein SAR1a (SAR1A) and the neuropeptide FF receptor 1 (NPFFR1). However, there is no evidence in the literature indicating their involvement with immune response. On SSC18, the marker rs 81468495 is located at position $31,646,160-31,693,830$. Downstream $114 \mathrm{~Kb}$ of this position, the MET proto-oncogene, that encodes the cell surface receptor for hepatocyte growth factor (HGF), is mapped. According to Chen et al. (1996) HGF and Met family genes may indeed be physiological regulators of monocyte-macrophage differentiation/maturation. Besides that, the MET gene was also associated to the immune regulation in humans with autism (Campbell et al., 2006; Heuer et al., 2011). On the SSCX, no gene was found spanning a $100 \mathrm{~kb}$ upstream or downstream. It was not possible to search for positional candidate genes for the other three markers with unknown location. Nevertheless, the absence of candidate genes mapped on those genomic regions does not exclude the existence of functionally important elements controlling the immune response in swine. These results highlight the relevance to improve the porcine genome annotation, which would increase the amount of biological information that can be derived from genomic studies.

To explore the genetic components associated with the immune response, GWAS was also carried out using ELISA to detect antibodies to a highly genetically conserved NP protein. Using the categorical definition of immune status (responders/nonresponders), a region on SSC12 was associated with immune response to FLUAV vaccination. The associated markers on SSC12 were segregating together in the haplotype AA, which was observed in high frequency in pigs that responded to FLUAV. The opposite haplotype GG was observed in all non-responder pigs. This region harbors four annotated genes in the swine genome: transducer of ERBB2, 1 (TOB1), nucleoside diphosphate kinase 1 (NME1), nucleoside diphosphate kinase 2 (NME2), and signal transducer and activator of transcription 2 (STAT2).

The TOB gene is a member of the anti-proliferative family of proteins that controls cell cycle progression. This gene has been implicated with several cellular mechanisms such as T helper 17 (Th17) cell function, including its involvement with human immunity and its use as a potential biomarker and as a therapeutic target (Baranzini, 2014). Influenza vaccination is known to promote antigen-specific IgG and IgA antibody response and also the pro- 
duction of an antigen-specific Th17 cell, inducing an inflammatory process, increasing morbidity among animals without improving viral clearance following challenge (Maroof et al., 2014). Recent studies have indicated that TOB1 interacts with Th17 and IL-2, regulating their activity and also blocking the expression of cell cycle genes (Salerno et al., 2014). Therefore, TOB1 works as a negative modulator of Th17, acting directly in the immune response to vaccination.

The NME1 and NME2 genes are also located in the associated region of SSC12. NME1 is involved with cell proliferation, differentiation and development, signal transduction and $G$ protein-coupled receptor endocytosis. NME2 is a negative regulator of Rho activity. Inhibition of Rho kinase activity causes a decrease in apoptosis and enhanced proliferation of CD4 T cells, an important group of cells involved with immune system (Hasan et al., 2013). Interferon-activated, dimerized signal transducers and activators of transcription (STAT2) gene are latent cytoplasmic transcription factors that bind to importins via a non- classical nuclear localization signals (NLSs) and also regulates the expression of genes involved in host immune defense (Levy and Darnell, 2002). STAT2 deficient animals have been identified to have a reduced responsiveness to IFN and consequently are more susceptible to viral infection (Park et al., 2000), suggesting its critical role against viral infection. Influenza A virus nucleoprotein also contains a non-classical NLS that is recognized by the C-terminal NLS binding site, indicating a similar mode of action of STAT proteins, suggesting a preference to be targeted by viruses to evade antiviral responses. In a human study, STAT2 gene was highly associated with the IFN signaling pathways, having an increase in its expression three days after influenza vaccination (Nakaya et al., 2011). Poland and collaborators (Poland et al., 2014) have identified a down regulation of miR-590-5p affecting the IL2 , IL2RA and Ig genes in T and B cells in humans three days post influenza vaccination.

When the immune response to NP protein was tested as a quantitative trait, three regions were significantly associated with variability of immune response. The regions were located on SSC1, SSC7, and SSC15. The associated region on SSC1 harbors four annotated genes: PPIL6 (Peptidylprolyl isomerase cyclophilin-like 6), FIG4 (FIG4 phosphoinositide 5-phosphatase), DDO (D-aspartate oxidase), and FYN (Tyrosine-protein kinase).

PPIL6 has been previously associated with the immune system, but no direct involvement with vaccination has been shown (Andrus et al., 2012). In the literature, the function of FIG4 and DDO has not been directly associated with immune response, therefore they were not explored.

FYN is associated with humoral immune response (Chaimowitz et al., 2013). The production of antigen-specific antibodies and the immune memory require an interaction between $\mathrm{B}$ and $\mathrm{T}$ cells, where the IL-4 secreted by T cells are important for B cell survivability (Perlmutter et al., 1993; Yusuf et al., 2010). FYN is part of the tyrosine kinase family, expressed in different type of cells, including lymphocytes. This kinase interacts with $\mathrm{B}$ and $\mathrm{T}$ cell receptors (BCR and TCR) and its deficiency impacts directly in the IL-4 signaling pathway (Latour et al., 2003). This will consequently affect the production of $\operatorname{IgG} 1$, IgE, and $\operatorname{IgG} 2 \mathrm{c}$, with a delayed antigen-specific $\operatorname{IgG} 1$ and IgG2b production, and decrease in antigen-specific IgG2c following immunization with a T-dependent antigen (Chaimowitz et al., 2013). FYN is also part of the same T cell signaling pathway involved with the MHC complex and the STAT gene. In this study, the genes STAT2, TOB1, and FYN have been identified as positional and functional candidate genes involved with immune response to vaccination as their function have been previously identified as a key component of the host antiviral response.

No annotated genes to the swine genome were identified in the regions associated with immune response on SSC7 and SSC15. However, when we expanded our search to $100 \mathrm{~Kb}$ upstream and
$100 \mathrm{~Kb}$ downstream of the associated markers, we identified the presence of the G protein-coupled receptor 110 (GPR110) gene on SSC7. The G protein-coupled receptors are highly conserved among vertebrates, and they have been reported to be involved with cellular response to immunological challenges in humans (Liebscher et al., 2011). Most mammalian cells have the ability to express different types of class I phosphatidylinositide 3-kinase isoforms, which are used by tyrosine kinases and the GPCRs to generate second messengers signaling molecules that are highly expressed in cells of the immune system. In addition, phosphatidylinositide 3-kinase isoforms have been shown to participate directly in the signaling pathway involved with immune cell development (Okkenhaug, 2013). Our GWAS has shown markers in the G-protein couple receptors and tyrosine kinases associated with the development of acquired immunity in piglets experimentally vaccinated with an in house vaccine to FLUAV.

At the light of the results presented here, the genomic regions and prospected candidate genes identified should be further investigated using other methodologies such as target resequencing to improve the knowledge on immune response to vaccination. Moreover, the associated SNPs found here should be validated in different populations to be used as genetic markers to select animals with better response to vaccination.

\section{Conclusions}

The results presented here further our knowledge on the genetic basis involved in the immune response to influenza vaccination in pigs. However, these results were obtained using a whole inactivated influenza virus vaccine adjuvanted with oil-in-water emulsion, and, consequently, they cannot be generalized for other vaccine formulations. The understanding of the genetic mechanisms involved in the response to vaccination will help to improve the efficacy of vaccines, preventing and controlling the spread of this zoonotic agent, reducing losses caused by its infection. The seven genomic regions associated with response to FLUAV vaccination found in this study, using HI and ELISA, provide new insights for vaccine development and efficacy. Furthermore, selection for animals with a better response to vaccination might be an important step to control and prevent infectious diseases in the livestock sector.

\section{Competing interests}

The authors declare that they have no competing interests. Mention of trade names or commercial products in this article is solely for the purpose of providing specific information and does not imply recommendation or endorsement by the EMBRAPA, Brazilian Agriculture Research Corporation.

\section{Acknowledgements}

The authors sincerely appreciate the technical assistance of Marisete F. Schiochet in the serological assays, Vanessa Haach in sampling collection and Dr. Jack Dekkers for the experimental design discussion. R. Zanella was supported by a BJT grant 373167/2012-1 from the National Council for Scientific and Technological Development (CNPq), Brazil. Funding was partially provided by the Brazilian Agricultural Research Corporation EMBRAPA (project number 02.11.01.006.00.07).

\section{References}

Andrus, B.M., Blizinsky, K., Vedell, P.T., Dennis, K., Shukla, P.K., Schaffer, D.J., Radulovic, J., Churchill, G.A., Redei, E.E., 2012. Gene expression patterns in the 
hippocampus and amygdala of endogenous depression and chronic stress models. Mol. Psychiatry 17, 49-61.

Badke, Y.M., Bates, R.O., Ernst, C.W., Schwab, C., Fix, J., Van Tassell, C.P., Steibel, J.P. 2013. Methods of tagSNP selection and other variables affecting imputation accuracy in swine. BMC Genet. 14, 8.

Baranzini, S.E., 2014. The role of antiproliferative gene Tob1 in the immune system. Clin. Exp. Neuroimmunol. 5, 132-136.

Bennett, R., 2003. The direct costs of livestock disease: the development of a system of models for the analysis of 30 endemic livestock diseases in Great Britain. J. Agric. Econ. 54, 55-71.

Bennett, R., Ijpelaar, J., 2005. Updated estimates of the costs associated with thirty four endemic livestock diseases in Great Britain: a note. J. Agric. Econ. 56, $135-144$.

Campbell, D.B., Sutcliffe, J.S., Ebert, P.J., Militerni, R., Bravaccio, C., Trillo, S., Elia, M. Schneider, C., Melmed, R., Sacco, R., Persico, A.M., Levitt, P., 2006. A genetic variant that disrupts MET transcription is associated with autism. Proc. Natl. Acad. Sci. U. S. A. 103, 16834-16839.

Carragher, D.M., Kaminski, D.A., Moquin, A., Hartson, L., Randall, T.D., 2008. A novel role for non-neutralizing antibodies against nucleoprotein in facilitating resistance to influenza virus. J. Immunol. 181, 4168-4176.

CDC, 2015. http://www.cdc.gov/media/releases/2015/s0226-acip.html (access: 08.28.15.).

Chaimowitz, N.S., Falanga, Y.T., Ryan, J.J., Conrad, D.H., 2013. Fyn kinase is required for optimal humoral responses. PLoS One 8, e60640.

Chen, Q., DeFrances, M.C., Zarnegar, R., 1996. Induction of met proto-oncogene (hepatocyte growth factor receptor) expression during human monocyte-macrophage differentiation. Cell Growth Differ. 7, 821-832.

Cox, R.J., Brokstad, K.A., Ogra, P., 2004. Influenza virus: immunity and vaccination strategies. Comparison of the immune response to inactivated and live, attenuated influenza vaccines. Scand J. Immunol. 59, 1-15.

Del Giudice, G., Rappuoli, R., 2015. Inactivated and adjuvanted influenza vaccines. Curr Top Microbiol. Immunol. 386, 151-180.

Detmer, S.E., Gunvaldsen, R.E., Harding, J.C., 2013. Comparison of influenza A virus infection in high- and low-birth-weight pigs using morphometric analysis. Influenza Other Respir Viruses 7 (Suppl. 4), 2-9.

Gauger, P.C., Vincent, A.L., Loving, C.L., Lager, K.M., Janke, B.H., Kehrli Jr., M.E., Roth J.A., 2011. Enhanced pneumonia and disease in pigs vaccinated with an inactivated human-like (delta-cluster) H1N2 vaccine and challenged with pandemic 2009H1N1 influenza virus. Vaccine 29, 2712-2719.

Glass, E.J., 2004. Genetic variation and responses to vaccines. Anim. Health Res. Rev. 5, 197-208.

Goodell, C.K., Prickett, J., Kittawornrat, A., Johnson, J., Zhang, J., Wang, C., Zimmerman, J.J., 2014. Evaluation of screening assays for the detection of influenza A Virus serum antibodies in swine. Transbound. Emerg. Dis.

Hasan, Z., Palani, K., Zhang, S., Lepsenyi, M., Hwaiz, R., Rahman, M., Syk, I., Jeppsson, B., Thorlacius, H., 2013. Rho kinase regulates induction of T-cell immune dysfunction in abdominal sepsis. Infect. Immun. 81, 2499-2506.

Heuer, L., Braunschweig, D., Ashwood, P., Van de Water, J., Campbell, D.B., 2011. Association of a MET genetic variant with autism-associated maternal autoantibodies to fetal brain proteins and cytokine expression. Transl. Psychiatry 1, e48.

Hoft, D.F., Babusis, E., Worku, S., Spencer, C.T., Lottenbach, K., Truscott, S.M., Abate, G., Sakala, I.G., Edwards, K.M., Creech, C.B., Gerber, M.A., Bernstein, D.I., Newman, F., Graham, I., Anderson, E.L., Belshe, R.B., 2011. Live and inactivated influenza vaccines induce similar humoral responses, but only live vaccines induce diverse T-cell responses in young children. J. Infect. Dis. 204, $845-853$.

Kelm, S.C., Freeman, A.E., Kehrli Jr., M.E., 2001. Genetic control of disease resistance and immunoresponsiveness. Vet. Clin. North Am. Food Anim. Pract. 17, 477-493.

Kich, J.D., Uthe, J.J., Benavides, M.V., Cantao, M.E., Zanella, R., Tuggle, C.K., Bearson, S.M., 2014. TLR4 single nucleotide polymorphisms (SNPs) associated with Salmonella shedding in pigs. J. Appl. Genet. 55, 267-271.

Kimman, T.G., Vandebriel, R.J., Hoebee, B., 2007. Genetic variation in the response to vaccination. Community Genet. 10, 201-217.

Kitikoon, P., Gauger, P.C., Vincent, A.L., 2014. Hemagglutinin inhibition assay with swine sera. Methods Mol. Biol. 1161, 295-301.

Larsen, D.L., Karasin, A., Zuckermann, F., Olsen, C.W., 2000. Systemic and mucosal immune responses to H1N1 influenza virus infection in pigs. Vet. Microbiol. $74,117-131$

Latour, S., Roncagalli, R., Chen, R., Bakinowski, M., Shi, X., Schwartzberg, P.L. Davidson, D., Veillette, A., 2003. Binding of SAP SH2 domain to FynT SH3 domain reveals a novel mechanism of receptor signalling in immune regulation. Nat. Cell Biol. 5, 149-154.

Levy, D.E., Darnell Jr., J.E., 2002. Stats: transcriptional control and biological impact. Nat. Rev. Mol. Cell Biol. 3, 651-662.

Liebscher, I., Muller, U., Teupser, D., Engemaier, E., Engel, K.M., Ritscher, L., Thor, D., Sangkuhl, K., Ricken, A., Wurm, A., Piehler, D., Schmutzler, S., Fuhrmann, H. Albert, F.W., Reichenbach, A., Thiery, J., Schoneberg, T., Schulz, A., 2011. Altered immune response in mice deficient for the G protein-coupled receptor GPR34. J. Biol. Chem. 286, 2101-2110.

Maroof, A., Yorgensen, Y.M., Li, Y., Evans, J.T., 2014. Intranasal vaccination promotes detrimental Th17-mediated immunity against influenza infection. PLoS Pathog. 10, e1003875.

Masic, A., Lu, X., Li, J., Mutwiri, G.K., Babiuk, L.A., Brown, E.G., Zhou, Y., 2010. Immunogenicity and protective efficacy of an elastase-dependent live attenuated swine influenza virus vaccine administered intranasally in pigs. Vaccine 28, 7098-7108

Meeusen, E.N., Walker, J., Peters, A., Pastoret, P.P., Jungersen, G., 2007. Current status of veterinary vaccines. Clin. Microbiol. Rev. 20, 489-510, table of contents.

Miller, R.S., Farnsworth, M.L., Malmberg, J.L., 2013. Diseases at the livestock-wildlife interface: status, challenges, and opportunities in the United States. Prev. Vet. Med. 110, 119-132.

Mitchell, V., Dietz, V.J., Okwo-Bele, J.M., Cutts, F.T., 2013. Immunization in developing countries. In: Plotkin, S.A., Orenstein, W.A., Offit, P.A. (Eds.) Vaccines. ,6th ed. Elsevier Saunders, pp. 1369-1394.

Moreno, C.R., Lantier, F., Berthon, P., Gautier-Bouchardon, A.V., Boivin, R., Lantier, I., Brunel, J.C., Weisbecker, J.L., Francois, D., Bouix, J., Elsen, J.M., 2003. Genetic parameters for resistance to the Salmonella abortusovis vaccinal strain Rv6 in sheep. Genet. Sel. Evol. 35, 199-217.

Nakaya, H.I., Wrammert, J., Lee, E.K., Racioppi, L., Marie-Kunze, S., Haining, W.N., Means, A.R., Kasturi, S.P., Khan, N., Li, G.M., McCausland, M., Kanchan, V., Kokko, K.E., Li, S., Elbein, R., Mehta, A.K., Aderem, A., Subbarao, K., Ahmed, R., Pulendran, B., 2011. Systems biology of vaccination for seasonal influenza in humans. Nat. Immunol. 12, 786-795

Nelson, M.I., Schaefer, R., Gava, D., Cantao, M.E., Ciacci-Zanella, J.R., 2015. Influenza A Viruses of human origin in swine, Brazil. Emerg. Infect. Dis. 21, 1339-1347.

Newport, M.J., Goetghebuer, T., Weiss, H.A., Whittle, H., Siegrist, C.A., Marchant, A., MRC Gambia Twin Study Group, 2004. Genetic regulation of immune responses to vaccines in early life. Genes Immun. 5, 122-129.

Okkenhaug, K., 2013. Rules of engagement: distinct functions for the four class I PI3K catalytic isoforms in immunity. Ann. N. Y. Acad. Sci. 1280, 24-26.

Olsen, C.W., Brammer, L., Easterday, B.C., Arden, N., Belay, E., Baker, I., Cox, N.J., 2002. Serologic evidence of H1 swine Influenza virus infection in swine farm residents and employees. Emerg. Infect. Dis. 8, 814-819.

Park, C., Li, S., Cha, E., Schindler, C., 2000. Immune response in Stat2 knockout mice. Immunity 13, 795-804.

Perez-Enciso, M., Misztal, I., 2011. Qxpak. 5: old mixed model solutions for new genomics problems. BMC Bioinf. 12, 202

Perlmutter, R.M., Levin, S.D., Appleby, M.W., Anderson, S.J., Alberola-Ila, J., 1993. Regulation of lymphocyte function by protein phosphorylation. Annu. Rev. Immunol. 11, 451-499.

Poland, G.A., Ovsyannikova, I.G., Kennedy, R.B., Lambert, N.D., Kirkland, J.L., 2014. A systems biology approach to the effect of aging, immunosenescence and vaccine response. Curr. Opin. Immunol. 29, 62-68.

Purcell, S., Neale, B., Todd-Brown, K., Thomas, L., Ferreira, M.A., Bender, D., Maller, J., Sklar, P., de Bakker, P.I., Daly, M.J., Sham, P.C., 2007. PLINK: a tool set for whole-genome association and population-based linkage analyses. Am. J. Hum. Genet. 81, 559-575.

Rajao, D.S., Anderson, T.K., Gauger, P.C., Vincent, A.L., 2014. Pathogenesis and vaccination of influenza A virus in swine. Curr. Top Microbiol. Immunol. 385, 307-326.

Rajao, D.S., Costa, A.T., Brasil, B.S., Del Puerto, H.L., Oliveira, F.G., Alves, F., Braz, G.F., Reis, J.K., Guedes, R.M., Lobato, Z.I., Leite, R.C., 2013. Genetic characterization of influenza virus circulating in Brazilian pigs during 2009 and 2010 reveals a high prevalence of the pandemic H1N1 subtype. Influenza Other Respir Viruses 7, 783-790.

Reed, L.J., Muench, H., 1938. A simple method of estimating fifty percent endpoints. Am. J. Hyg. 27, 493-497.

Salerno, F., van Lier, R.A., Wolkers, M.C., 2014. Better safe than sorry: TOB1 employs multiple parallel regulatory pathways to keep Th17 cells quiet. Eur. J. Immunol. 44, 646-649.

Sandbulte, M.R., Platt, R., Roth, J.A., Henningson, J.N., Gibson, K.A., Rajao, D.S., Loving, C.L., Vincent, A.L., 2014. Divergent immune responses and disease outcomes in piglets immunized with inactivated and attenuated H3N2 swine influenza vaccines in the presence of maternally-derived antibodies. Virology 464-465, 45-54

Schaefer, R., Ciacci-Zanella, J.R., Brentano, L., Vincent, A.L., Ritterbusch, G.A., Silveira, S., Caron, L., Mores, N., 2011. Isolation and characterization of a pandemic H1N1 influenza virus in pigs in Brazil. Pesquisa Veterinaria Bras. 31, $761-767$.

Schaefer, R., Rech, R.R., Gava, D., Cantao, M.E., da Silva, M.C., Silveira, S., Zanella, J.R. 2015. A human-like H1N2 influenza virus detected during an outbreak of acute respiratory disease in swine in Brazil. Arch. Virol. 160, 29-38.

Settles, M., Zanella, R., McKay, S.D., Schnabel, R.D., Taylor, J.F., Whitlock, R., Schukken, Y., Van Kessel, J.S., Smith, J.M., Neibergs, H., 2009. A whole genome association analysis identifies loci associated with Mycobacterium avium subsp. paratuberculosis infection status in US holstein cattle. Anim. Genet. 40 655-662.

Sitte, K., Brinkworth, R., East, I.J., Jazwinska, E.C., 2002. A single amino acid deletion in the antigen binding site of BoLA-DRB3 is predicted to affect peptide binding. Vet. Immunol. Immunopathol. 85, 129-135.

Tan, P.L., Jacobson, R.M., Poland, G.A., Jacobsen, S.J., Pankratz, V.S., 2001. Twin studies of immunogenicity-determining the genetic contribution to vaccine failure. Vaccine 19, 2434-2439.

Team, R.D.C., 2009. R: A Language and Environment for Statistical Computing. Foundation for Statistical Computing, Vienna, Austria.

Van Loveren, H., Van Amsterdam, J.G., Vandebriel, R.J., Kimman, T.G., Rumke, H.C., Steerenberg, P.S., Vos, J.G., 2001. Vaccine-induced antibody responses as parameters of the influence of endogenous and environmental factors. Environ. Health Perspect. 109, 757-764. 
Van Reeth, K., Brown, I.H., Olsen, C.W., 2012. Influenza Virus. In: Zimmerman, J.J., Karriker, L.A., Ramirez, A., Schwartz, K.J., Stevenson, G.W. (Eds.), Diseases of Swine., 10th ed. Iowa State University Press, Ames, pp. 557-571.

Wellcome Trust Case Control, C., 2007. Genome-wide association study of 14,000 cases of seven common diseases and 3000 shared controls. Nature 447, 661-678.

Yusuf, I., Kageyama, R., Monticelli, L., Johnston, R.J., Ditoro, D., Hansen, K., Barnett, B., Crotty, S., 2010. Germinal center T follicular helper cell IL-4 production is dependent on signaling lymphocytic activation molecule receptor (CD150).J. Immunol. 185, 190-202.

Zanella, R., Settles, M.L., McKay, S.D., Schnabel, R., Taylor, J., Whitlock, R.H., Schukken, Y., Van Kessel, J.S., Smith, J.M., Neibergs, H.L., 2011. Identification of loci associated with tolerance to Johne's disease in Holstein cattle. Anim. Genet. 42, 28-38. 